\title{
Direct and Catalytic C-Glycosylation of Arenes: Expeditious Synthesis of the Remdesivir Nucleoside
}

Authors: Carla Obradors, $\uparrow$ Benjamin Mitschke, $\uparrow$ Miles H. Aukland, $\uparrow$ Markus Leutzsch, Oleg Grossmann, Sebastian Brunen, Sebastian A. Schwengers, Benjamin List*

\section{Affiliations:}

${ }^{1}$ Max-Planck-Institut für Kohlenforschung, Kaiser-Wilhelm-Platz 1, 45470, Mülheim an der Ruhr, Germany.

*Correspondence to: list@kofo.mpg.de

tThese authors contributed equally to this work.

\section{Abstract:}

Since early 2020, scientists have strived to find an effective solution to fight SARS-CoV-2, especially by developing reliable vaccines that inhibit the spread of the disease and repurposing drugs for combatting its effects on the human body. The antiviral prodrug remdesivir is still the most widely used therapeutic during the early stage of the infection. However, the current synthetic routes rely on the use of protecting groups, air-sensitive reagents, and cryogenic conditions, impeding the cost-efficient supply to patients. We therefore focused on the development of a straightforward, direct addition of (hetero)arenes to unprotected sugars. Here we report a silylium-catalyzed and completely stereoselective C-glycosidation that initially yields the open-chain polyols, which can be selectively cyclized to provide either the kinetic $\alpha$-furanose or the thermodynamically favored $\beta$-anomer. The method significantly expedites the synthesis of remdesivir precursor GS441524 after subsequent $\mathrm{Mn}$-catalyzed $\mathrm{C}-\mathrm{H}$ oxidation and deoxycyanation.

\section{Main Text:}

More than a year after COVID-19 spread worldwide, humankind still strives for stable recovery. Nevertheless, we have witnessed the striking effect of applied and basic science on restraining the impact of SARS-CoV-2. This outbreak has been tackled especially intensely from the pharmaceutical sector in terms of small-molecule therapeutics, vaccinations and biomedical devices. The pandemic has also driven a global effort to repurpose existing drugs to enable a rapid and targeted response. ${ }^{1}$ Remdesivir (Veklury) is an antiviral prodrug that was originally evaluated in clinical trials to fight the Ebola virus $^{2,3}$ and, although showing somewhat mixed results against COVID-19, it is still the most widely used treatment during the early stage of infection. ${ }^{4}$ In general, nucleotide analogs such as remdesivir are a class of compounds which are prominent for their RNA antiviral properties (Figure 1A)., ${ }^{5,6}$ Besides the ubiquitous presence of the monosaccharide derivative, their synthesis still relies on multi-step sequences and the use of protecting groups due to the selectivity challenges inherent in the functionalization of unprotected sugars. ${ }^{7,8}$ In the case of remdesivir, Gilead's industrial route starts with the perbenzylated D-ribonolactone (Figure 1B) - which is obtained in four steps from D-ribose - and requires sensitive reagents and cryogenic conditions for several of the steps involved. ${ }^{9}$ This context of necessity has also inspired the organic chemistry community and embedded a spur of novel synthetic methodologies, such as new approaches to build the heterocyclic arene ${ }^{10,11}$ and improvements to the organocatalytic asymmetric phosphoramidation to introduce the monophosphate-like side chain. ${ }^{12}$ However, efforts towards streamlining the construction of the core nucleoside itself have remained elusive. ${ }^{13}$ Recent advancements for the direct assembly of nucleosides have focused on the formation of a $\mathrm{C}$-heteroatom bond in the anomeric position, ${ }^{14}$ whereas examples for the $\mathrm{C}-\mathrm{C}$ disconnection are scarce ${ }^{15,16}$ We aimed at the development of a new and greatly facilitated route to remdesivir featuring the direct arylation of D-ribose by means of silylium catalysis as the key step (Figure 1C). Here we report the completely regio- and stereoselective addition of (hetero)arenes to unprotected sugars, which in turn furnishes the nucleoside intermediate in a one-pot fashion. The divergent formation of the $\alpha$ - or the $\beta$-anomer is achieved either by kinetic control or through a thermodynamically-driven epimerization, respectively. ${ }^{17}$ Finally, the new method unlocks an expedient synthesis of the remdesivir precursor GS441524 by virtue of a selective $\mathrm{Mn}$-catalyzed benzylic $\mathrm{C}-\mathrm{H}$ oxidation and diastereoselective deoxycyanation. 
A Examples of Antiviral Nucleosides

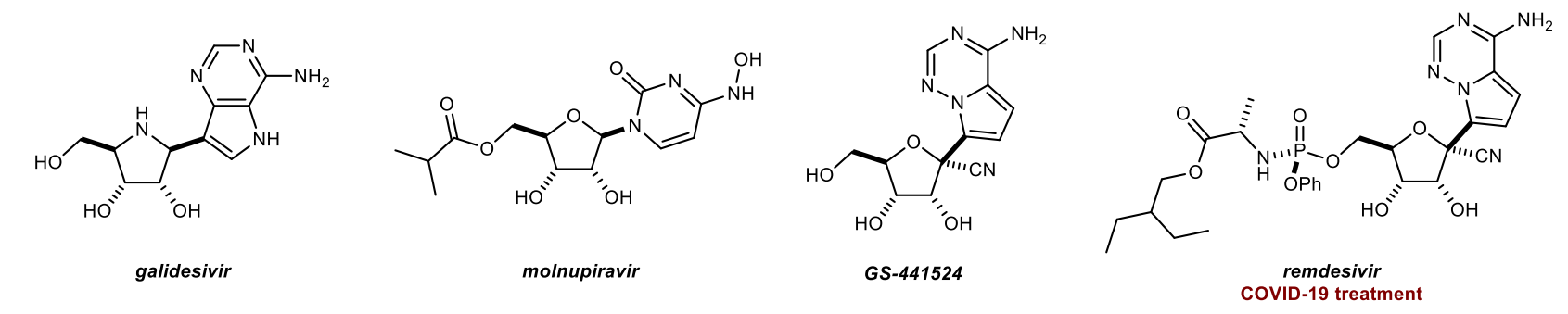

B Established Multi-Step Synthetic Route

C Newly Designed Route
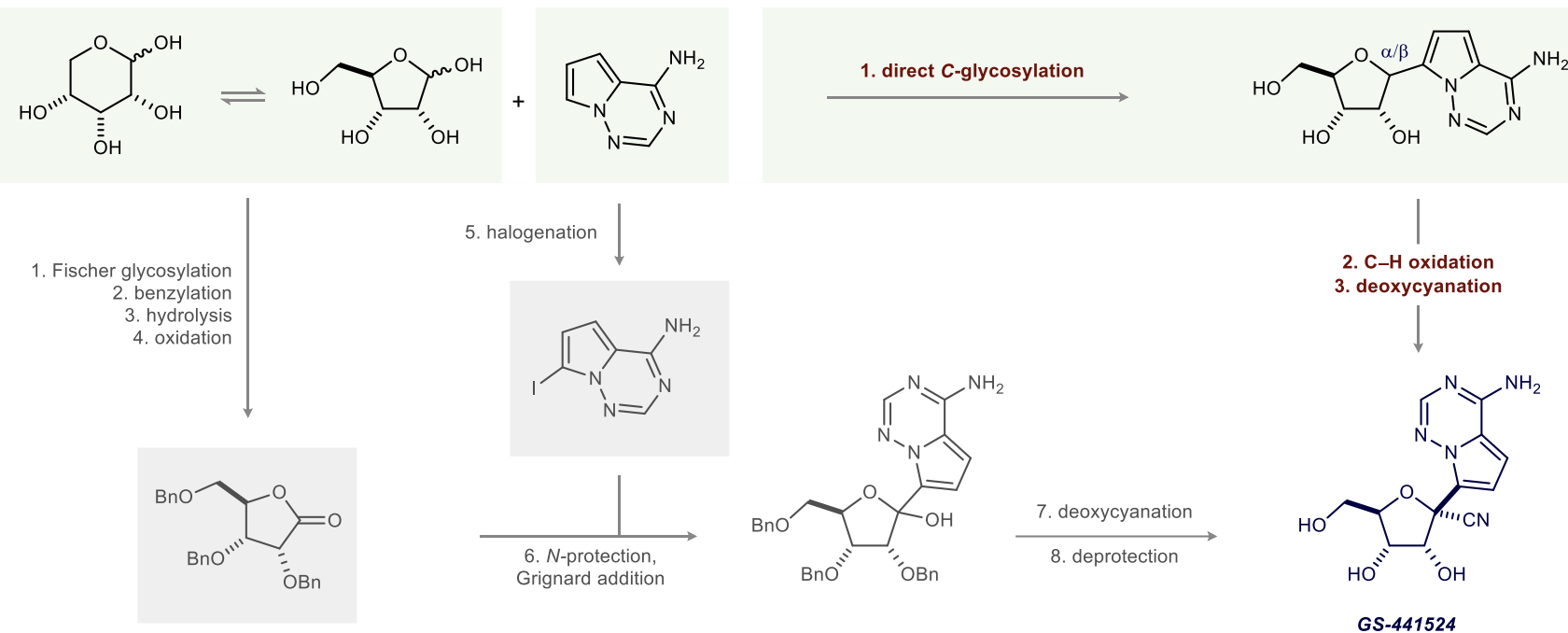

Figure 1. A. Examples of nucleoside-based antivirals. B. Established sequence towards the synthesis of remdesivir. C. Design here: direct arylation of free D-ribose.

Our approach harnesses the native nucleophilicity of the heterocycle, which eliminates the requirement for prefunctionalization and the preparation of the air-sensitive organometallic reagent. Furthermore, we exploit a Lewis acidcatalyzed transient silylation of the carbohydrate in order to concurrently fulfill two main purposes: in situ protection of all protic functionalities as well as selective anomeric activation (Figure 2A). We found that D-ribose is rapidly activated by catalytic TMSOTf at $25{ }^{\circ} \mathrm{C}$, which is regenerated upon protodesilylation of the innocent silicon source $\mathrm{N}, \mathrm{O}$ bis(trimethylsilyl)trifluoroacetamide (BSTFA). The key $\mathrm{C}-\mathrm{C}$ bond formation occurs at $50{ }^{\circ} \mathrm{C}$ under neat conditions leading exclusively to a single linear isomer $1-\mathrm{TMS}_{6}$ in quantitative yield. This selectivity is quite remarkable when taking into consideration the complex structure of free D-ribose, which consists of a mixture of five isomers: the $\alpha$ - and $\beta$-pyranose and -furanose, as well as the open form. ${ }^{18,19}$ However, during the transformation this dynamic mixture converges diastereoselectively to a single product. Subsequent desilylation of the crude reaction mixture with TBAF delivers the linear analog 1 in a one-pot fashion (86\%), rendering the use of silicon traceless. Preliminary attempts have revealed that the method is also competent for the functionalization of alternative sugars and (hetero)arenes (Figure 2C); for instance, reaction with D-xylose also delivers product 2 as a single diastereoisomer in slightly lower yield (53\%). Moreover, addition of an indole derivative occurs in good yield at $25^{\circ} \mathrm{C}$ to polyol $3(69 \%)$. Finally, 1,3,5-trimethoxybenzene similarly enables the derivatization to compound 4 in $71 \%$ yield. 
A Direct and Selective C-Glycosylation of Ribose

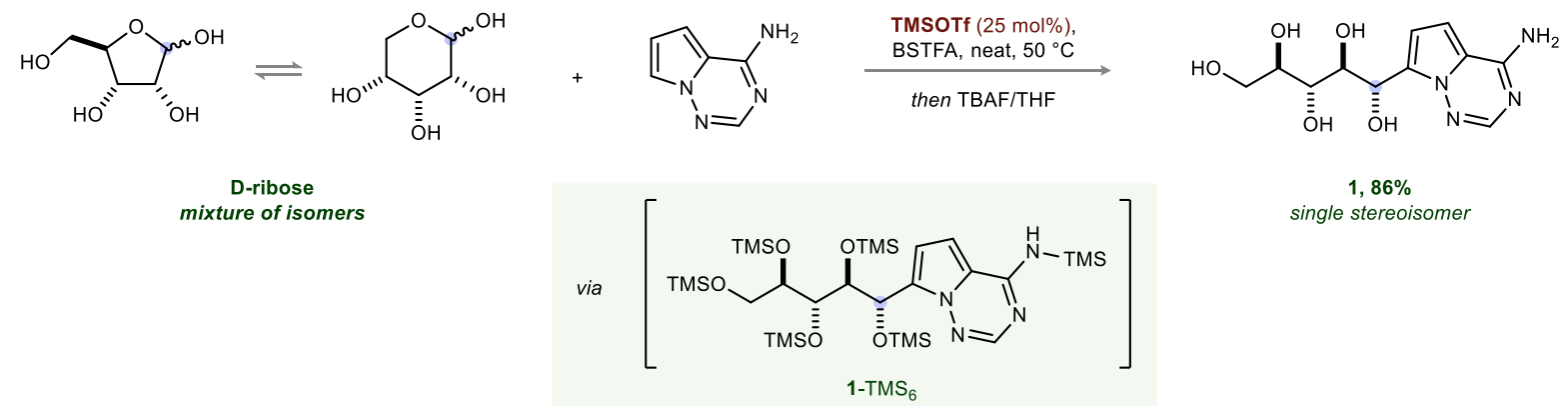

B Diastereoconvergent Silylation as the Basis for Diastereoselective C-C Bond Formation

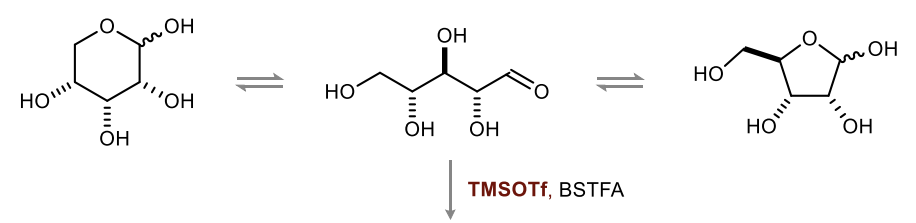
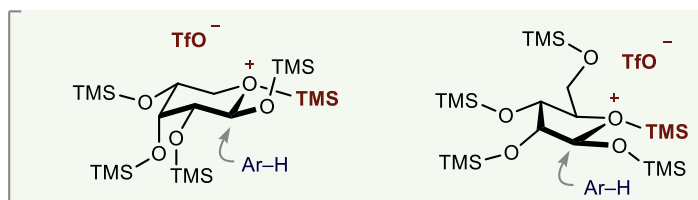

$\downarrow \mathrm{Ar}-\mathrm{H}$

single product stereoisomer

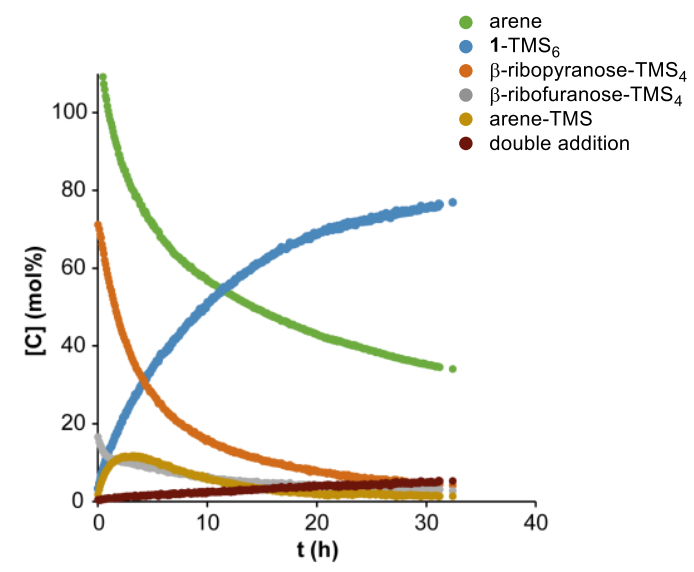

C Derivatization of Other Sugars with Other (Hetero)Arenes

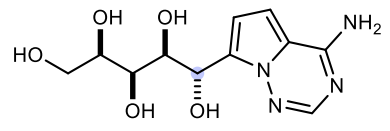

$2,53 \%$

from $D$-xylose single stereoisomer

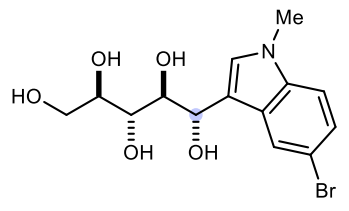

$3,69 \%$

from $D$-ribose single stereoisomer

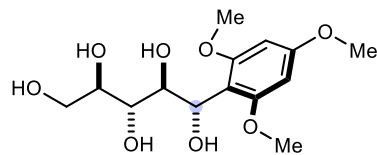

$4,71 \%$

from $D$-ribose single stereoisomer

Figure 2. A. Diastereoselective functionalization of D-ribose by transient silylation. B. Mechanistic insights on the $\mathrm{C}-\mathrm{C}$ bond formation. C. Additional examples of other sugars with other (hetero)arenes.

Lewis acid-catalyzed nucleophilic additions to carbohydrate derivatives are generally believed to proceed via oxocarbenium intermediates, derived from the activation of the anomeric substituent. ${ }^{20-22}$ However, this assumption is questioned here in light of the formation of a single linear product. Instead, we propose a mechanism in which TMSOTf coordinates to the endocyclic oxygen atom of the sugar prior to reaction with the heterocycle leading to ring opening (Figure 2B). ${ }^{23}$ Thorough NMR analysis confirmed the silylation of the sugar at $25{ }^{\circ} \mathrm{C}$, delivering the TMS-protected ribopyranose and -furanose in $77 \%$ and $21 \%$ yield, respectively (see Supporting Information, Figure S10-23). Only the $\beta$ anomers are present for both intermediates, in which the anomeric groups are oriented trans to the vicinal siloxy group for steric reasons (see Supporting Information, Figure S46 for DFT calculations). Consequently, successive nucleophilic substitution via an $\mathrm{S}_{\mathrm{N}} 2$-type mechanism converges to the product as a single diastereoisomer. Kinetic analysis at $50{ }^{\circ} \mathrm{C}$ revealed that consumption of the arene mirrors the formation of the product, with the pyranose isomer reacting faster than the furanose. Traces of double addition were also detected. 


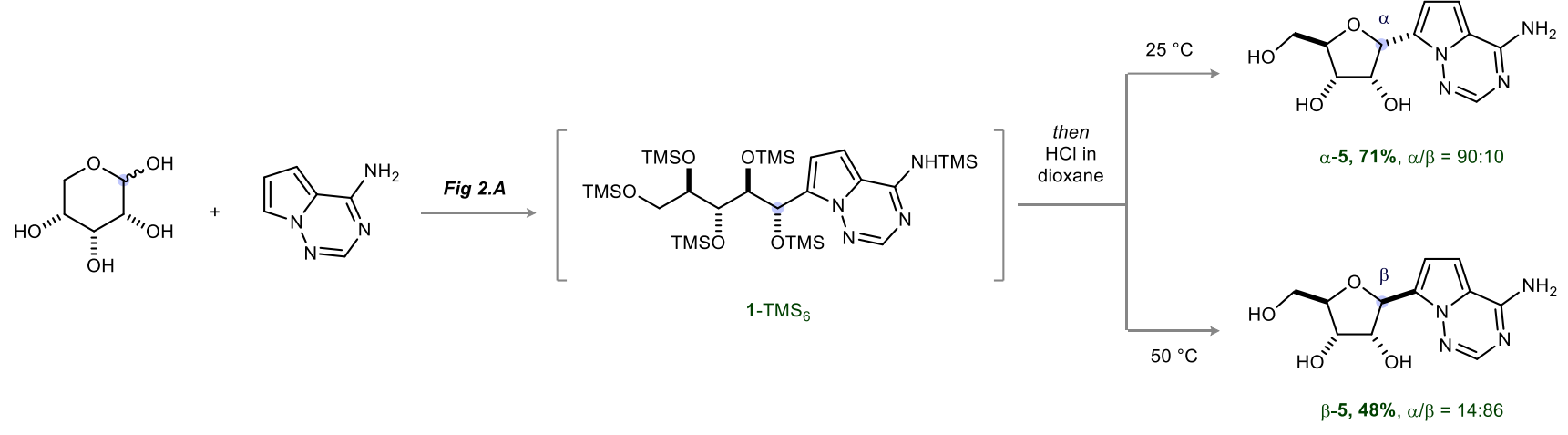

Figure 3. Selective syntheses of both $\alpha$ - and $\beta$ - $C$-glycosylated nucleosides by variation of cyclization temperature.

Remarkably, we found that when the reaction mixture was subjected to acid treatment rather than to fluoride, the corresponding $C$-ribofuranosylated products are obtained (Figure 3). Formation of the $\alpha$-product $\mathbf{5}$ is achieved by performing the acid treatment at $25^{\circ} \mathrm{C}$ with good yield and high diastereoselectivity $(71 \%, \alpha / \beta=90: 10)$. In contrast, acid treatment at $50^{\circ} \mathrm{C}$ leads directly to the thermodynamically favoured $\beta$-nucleoside $5(48 \%, \alpha / \beta=14: 86)$. Epimerization from $\alpha$ - to $\beta$-could be monitored by ${ }^{1} \mathrm{H}$ NMR after resubmission of isolated product $\alpha-5$ to $\mathrm{HCl}$ in dioxane at $50{ }^{\circ} \mathrm{C}$ (see Supporting Information, Figure S27).

In the context of the synthesis of the antiviral drug GS-441524, subsequent oxidative installation of the nitrile moiety was required. Thus, we envisioned an initial $\mathrm{C}-\mathrm{H}$ oxidation to furnish the stereolabile hemiacetal 7 (Figure 4), which could in turn be diastereoconvergently deoxycyanated to provide GS-441524.

After investigating distinct approaches, the desired reactivity was achieved by means of a Mn-catalyzed benzylic $\mathrm{C}-\mathrm{H}$ oxidation. ${ }^{24}$ Notably, exclusive reactivity of the $\alpha$-anomer $\mathbf{5}$ was observed, most likely resulting from the higher accessibility of its $\beta$-hydrogen atom to the Mn-porphyrin complex, in accordance with DFT-optimized ground-state structures (see Supporting Information, Figure S31). Shorter reaction times and portionwise addition of the oxidant are crucial factors determining the reaction outcome, with fragmentative overoxidation to the ribonolactone being the most prominent side reaction. Ultimately, conversion as well as selectivity were enhanced by precipitation of the polar $\mathrm{C}-\mathrm{H}$ oxidation product from the solvent mixture ( $n$-pentane $/ \mathrm{CH}_{2} \mathrm{Cl}_{2} 5: 1 \mathrm{v} / \mathrm{v}$ ). Starting from peracetylated $\alpha$-ribonucleoside 6, which was synthesized on a $15 \mathrm{mmol}$ scale in $60 \%$ yield from D-ribose with a single purification via column chromatography, oxidation product 7 was obtained as an inconsequential mixture of isomers (equilibrium distribution in $\mathrm{CD}_{2} \mathrm{Cl}_{2}: 46: 54$ ketone/hemiacetal, $\alpha: \beta=63: 37$ ) in $45 \%$ yield.

Previous reports on the deoxycyanation of the benzyl-protected hemiacetal provided great insight for the final step of our route. ${ }^{9}$ Due to the neighbouring acetoxy groups, however, intermediate $\mathbf{7}$ showed significantly lower reactivity. As a result, we substituted the original mixture of TfOH/TMSOTf with the even stronger Brønsted acid HNTf $\mathrm{H}_{2}$, which generates the active Lewis acid TMS-NTf 2 in situ upon treatment with TMSCN. In addition, reducing the equivalents of TMSCN prevents further reaction of the product (see Supporting Information, Table S3) and overnight reaction at $-40{ }^{\circ} \mathrm{C}$ delivered the peracetylated cyanation product in $84 \%$ yield and with good diastereoselectivity ( $\alpha: \beta=87: 13$ ). Notably, GS-441524 precipitated as a single stereoisomer during the subsequent methanolysis in $81 \%$ yield, $68 \%$ from hemiacetal 7 . Overall, this approach greatly improves access to the target compound through just three steps requiring purification and with a total yield of $18 \%$ from D-ribose; in contrast to the existing process that delivers GS-441524 in 7 steps (longest linear sequence) and with $12 \%$ yield (see Supporting Information, p. 45 ). 


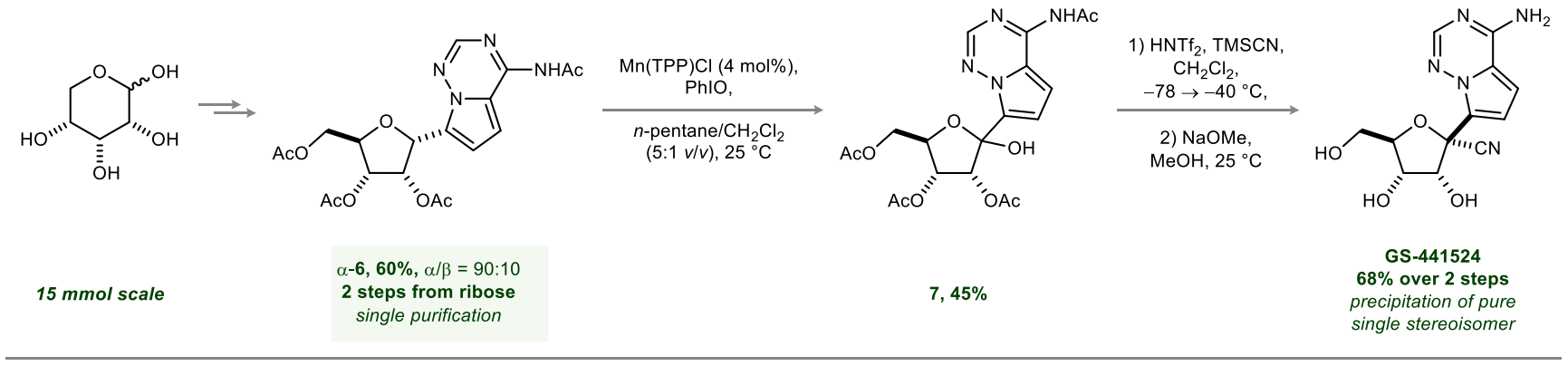

Figure 4. Benzylic $\mathrm{C}-\mathrm{H}$ oxidation and diastereoselective deoxycyanation of peracetylated ribonucleoside $\alpha-6$.

We have designed and developed a more practical and efficient formal synthesis of remdesivir starting from free D-ribose. Our route features a novel and completely stereoselective nucleophilic addition of (hetero)arenes to carbohydrates without pre-installation of any protecting or activating groups. The process delivers the linear products via silylium catalysis diastereoselectively due to convergent silylation towards the TMS-protected $\beta$-ribopyranose and -furanose prior to the $\mathrm{C}-\mathrm{C}$ bond formation. The anomeric configuration of the $\mathrm{C}$-nucleoside is tuned simply by means of thermodynamic control during the acid-mediated cyclization. Benzylic $\mathrm{C}-\mathrm{H}$ oxidation followed by deoxycyanation indeed streamlines the existing chemical synthesis of GS-441524 from D-ribose and the artificial nucleobase.

\section{References and Notes:}

1. Savi, C. D., Hughes, D. L. \& Kvaerno, L. Quest for a COVID-19 Cure by Repurposing Small-Molecule Drugs: Mechanism of Action, Clinical Development, Synthesis at Scale, and Outlook for Supply. Org. Process Res. Dev. 24, $940-976$ (2020).

2. Warren, T. K. et al. Therapeutic efficacy of the small molecule GS-5734 against Ebola virus in rhesus monkeys. Nature 531, 381-385 (2016).

3. Siegel, D. et al. Discovery and Synthesis of a Phosphoramidate Prodrug of a Pyrrolo[2,1- $f$ ][triazin-4-amino] Adenine $C$ -Nucleoside (GS-5734) for the Treatment of Ebola and Emerging Viruses. J. Med. Chem. 60, 1648-1661 (2017).

4. Rezagholizadeh, A., Khiali, S., Sarbakhsh, P. \& Entezari-Maleki, T. Remdesivir for treatment of COVID-19; an updated systematic review and meta-analysis. Eur. J. Pharmacol. 897, 173926 (2021).

5. Štambaský, J., Hocek, M. \& Kočovský, P. C-Nucleosides: Synthetic Strategies and Biological Applications. Chem. Rev. 109, 6729-6764 (2009).

6. Eastman, R. T. et al. Remdesivir: A Review of Its Discovery and Development Leading to Emergency Use Authorization for Treatment of COVID-19. ACS Central Sci. (2020) doi:10.1021/acscentsci.0c00489.

7. Yang, Y. \& Yu, B. Recent Advances in the Chemical Synthesis of C-Glycosides. Chem. Rev. 117, 12281-12356 (2017).

8. Dimakos, V. \& Taylor, M. S. Site-Selective Functionalization of Hydroxyl Groups in Carbohydrate Derivatives. Chem. Rev. 118, 11457-11517 (2018).

9. Vieira, T. et al. Development of a Large-Scale Cyanation Process Using Continuous Flow Chemistry En Route to the Synthesis of Remdesivir. Org. Process Res. Dev. 24, 2113-2121 (2020).

10. Knapp, R. R., Tona, V., Okada, T., Sarpong, R. \& Garg, N. K. Cyanoamidine Cyclization Approach to Remdesivir's Nucleobase. Org. Lett. 22, 8430-8435 (2020). 
11. Paymode, D. J. et al. Expanding Access to Remdesivir via an Improved Pyrrolotriazine Synthesis: Supply Centered Synthesis. Org. Lett. 22, 7656-7661 (2020).

12. Wang, M. et al. Catalytic Asymmetric Synthesis of the anti-COVID-19 Drug Remdesivir. Angew. Chem. Int. Ed. 59, 20814-20819 (2020).

13. Xie, Y. et al. Weinreb Amide Approach to the Practical Synthesis of a Key Remdesivir Intermediate. J. Org. Chem. 86, 5065-5072 (2021).

14. Hanessian, S. \& Lou, B. Stereocontrolled Glycosyl Transfer Reactions with Unprotected Glycosyl Donors. Chem. Rev. $100,4443-4464$ (2000).

15. Sato, S., Akiya, T., Suzuki, T. \& Onodera, J. Environmentally friendly C-glycosylation of phloroacetophenone with unprotected d-glucose using scandium(III) trifluoromethanesulfonate in aqueous media: key compounds for the syntheses of mono- and di-C-glucosylflavonoids. Carbohyd. Res. 339, 2611-2614 (2004).

16. Chen, M. C. et al. Spontaneous Prebiotic Formation of a $\beta$-Ribofuranoside That Self-Assembles with a Complementary Heterocycle. J. Am. Chem. Soc. 136, 5640-5646 (2013).

17. Ren, R. X.-F., Chaudhuri, N. C., Paris, P. L., Rumney \& Kool, E. T. Naphthalene, Phenanthrene, and Pyrene as DNA Base Analogues: Synthesis, Structure, and Fluorescence in DNA. J. Am. Chem. Soc. 118, 7671-7678 (1996).

18. Šišak, D. et al. The Crystal Structure of D-Ribose-At Last! Angew. Chem. Int. Ed. 49, 4503-4505 (2010).

19. Drew, K. N., Zajicek, J., Bondo, G., Bose, B. \& Serianni, A. S. 13C-labeled aldopentoses: detection and quantitation of cyclic and acyclic forms by heteronuclear 1D and 2D NMR spectroscopy. Carbohyd. Res. 307, 199-209 (1998).

20. Bennek, J. A. \& Gray, G. R. An efficient synthesis of anhydroalditols and allylic-glycosides. J. Org. Chem. 52, 892-897 (1987).

21. Forsyth, C. J., Sabes, S. F. \& Urbanek, R. A. An Efficient Total Synthesis of Okadaic Acid. J. Am. Chem. Soc. 119, 83818382 (1997).

22. Bennua-Skalmowski, B., Krolikiewicz, K. \& Vorbrüggen, H. A new simple nucleoside synthesis. Tetrahedron Lett. 36, 7845-7848 (1995).

23. Zhang, J., Park, S. \& Chang, S. Selective C-O Bond Cleavage of Sugars with Hydrosilanes Catalyzed by Piers' Borane Generated In Situ. Angew. Chem. Int. Ed. 56, 13757-13761 (2017).

24. Gunay, A. \& Theopold, K. H. C-H Bond Activations by Metal Oxo Compounds. Chem. Rev. 110, 1060-1081 (2010).

Acknowledgements: This work has been a true group effort; we appreciate the early evaluation of alternative routes from Dr Santanu Ghosh, Manuel Scharf, Mathias Turberg, Dr Chandra Kanta De and Dr Tynchtyk Amatov. We also thank the rest of the group members for fruitful discussions: Dr Sayantani Das, Christian David Diaz-Oviedo, Dr Gabriela Gerosa, Joyce Grimm, Jennifer Kennemur, Yihang Li, Dr Rajat Maji, Dr Jie Ouyang, Dr Roberta Properzi, Vikas Kumar Singh, Dr Vijay Wakchaure, Dr Hui Zhou, Dr Chendan Zhu, Dr Pinglu Zhang, as well as the help of our technicians. We gratefully acknowledge the kind gift of the required pyrrolotriazine from Bayer AG (Dr Steffen Müller). We thank the MS and NMR departments of the Max-Planck-Institut für Kohlenforschung for analytics. Finally, generous support from the Deutsche Forschungsgemeinschaft (Leibniz Award to B. L. and Germany's Excellence Strategy-EXC 2033-390677874-RESOLV), the European Research Council (European Union's Horizon 2020 research and innovation program " $\mathrm{C}-\mathrm{H}$ Acids for Organic Synthesis, CHAOS" Advanced Grant Agreement No. 694228), the Alexander von Humboldt Foundation as well as the Bayer 
Science \& Education Foundation (Research Fellowship and sponsor to C. O.) and the Fonds der Chemischen Industrie (Kekulé Fellowship to B.M.) is gratefully acknowledged.

Author Contributions: $\mathrm{BL}, \mathrm{CO}, \mathrm{BM}$ and $\mathrm{MHA}$ conceived and directed the project. $\mathrm{CO}$ discovered the direct $C$-glycosylation discussed in this publication. CO, BM, MHA, OG and SB optimized the method. MHA, OG, and SAS performed the scale-up experiments. MHA and $\mathrm{CO}$ elaborated the scope. $\mathrm{CO}$ and $\mathrm{ML}$ conducted the mechanistic studies. BM developed the subsequent synthesis of GS-441524 and performed the DFT calculations. MHA, CO, OG and SB explored in parallel alternative routes. $\mathrm{CO}, \mathrm{BM}, \mathrm{MHA}$ and $\mathrm{BL}$ prepared the manuscript and Supporting Information.

Competing interests: A patent has been filed by the Max-Planck-Institut für Kohlenforschung covering the process and subsequent synthesis of GS-441524. 